\section{Socio-economic status of patients registered with NHS dental services in Scotland: an inverse 'dental' care law?}

\author{
C. M. Jones, ${ }^{* 1}$ D. Clouting ${ }^{2}$ and G. McCartney ${ }^{3}$
}

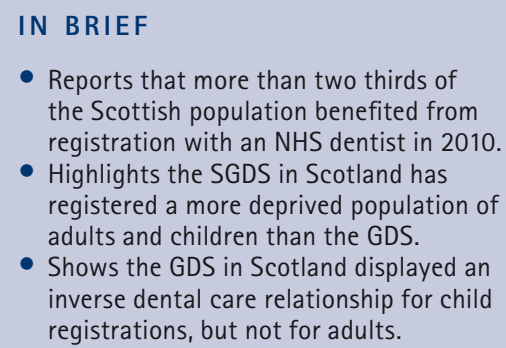

Objective To compare the socioeconomic characteristics of the population registered with an NHS dentist, split by the NHS General Dental Services (GDS) and Salaried General Dental Services (SGDS) across Scotland. Method Routine data on NHS dental registrations were obtained from the Information Services Division of NHS Scotland. Registrations by residential postcode ranked by Scottish Index of Multiple Deprivation (SIMD) quintiles were calculated separately for adults and children for both GDS and SGDS. Spearman's rank correlation coefficient and the relative index of inequality (RII) were calculated. Results In September 2010,69\% $(3,581,663)$ of the Scottish population was registered with an NHS dentist. The GDS had 95\% of the total registrations and 5\% were with the SGDS. There was a linear relationship in the SGDS where dental registrations with the SGDS were higher the more deprived the population of adults (RII = -1.6) and for children $(\mathrm{RII}=-7.8)$. In the GDS there was an inverse care relationship for children $(\mathrm{R} I=1.4)$ but the confidence intervals for the adults' RII included zero so was not significant. Total NHS dental registration across Scotland (for both children and adults) did not vary by SIMD quintile. Conclusion Contrary to expectations, no inverse dental care law could be demonstrated for adults receiving NHS dental care in Scotland in the GDS. There was a statistically significant inverse dental care law for children in the GDS. The SGDS has higher registration rates among the most deprived population and acts to reduce inequality in access to dental care, thereby removing any inverse dental care law.

\section{INTRODUCTION}

The inverse care law was proposed by a general medical practitioner, Julian TudorHart in $1971 ;$ ' the availability of good medical care tends to vary inversely with the need for it in the population served. This inverse care law operates more completely where medical care is most exposed to market forces, and less so where such exposure is reduced. The market distribution of medical care is a primitive and historically outdated social form, and any return to it would further exaggerate the maldistribution of medical resources'

Although originally applied to general medical services, it has been reported more

'NHS Health Scotland, Thistle House, 91 Haymarket Terrace, Edinburgh, EH12 5HE, Scotland; ${ }^{2}$ NHS Borders, Newstead, Melrose, TD6 9DA, Scotland; ${ }^{3}$ NHS Health Scotland, Elphinstone House, 65 West Regent Street, Glasgow, G2 2AF, Scotland

*Correspondence to: Colwyn M. Jones

Email: colwyn.jones@nhs.ne

Online article number E14

Refereed Paper - accepted 6 July 2012

DOI: 10.1038/sj.bdj.2013.223

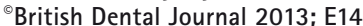

recently that the 'inverse care law' applies to UK NHS general dental services under the fee for item system. ${ }^{2}$

Previous reports showed the uneven distribution of dentists in the UK by wide variation in dentist/population ratios and a clear association between social class of a population and dentist/population ratios. ${ }^{3,4}$ From 1977 to 1995 , the distribution of general dental practitioners (GDPs) in England was reported to become more equitable, but that it bore little relation to levels of disease measured by $\mathrm{dmft}$ and $\mathrm{dt}$ in 5- and 12-year-old children. ${ }^{5}$

In 1985, Carmichael reported that the availability of dental care in the city of Newcastle-upon-Tyne was greater in more affluent areas and poorer in officially designated 'priority' areas. ${ }^{6}$ Reports have confirmed an inverse dental care law in both children $^{7,8}$ and adults. ${ }^{9}$ Interestingly Dyer et $a .^{9}$ in their health equity audit (HEA) approach, stated 'an overt 'inverse care law' did not exist for NHS services provided from dental practices in Sheffield' on the basis of the greater self-reported availability of dentists' time in practices in areas of higher socioeconomic deprivation (as approximated by levels of income support). However, no statistical test was offered and there was a two year time lag between the self-reported availability data and the earlier registration data. One finding from this paper, of a negative correlation between NHS registrations and deprivation at electoral ward level $(r=-0.59)$, suggesting an inverse care relationship, was not discussed.

Successive guidance has stressed the central role of the general dental practitioner as the preferred provider of routine dental services in Scotland. They are paid through a hybrid system of fee per item (pay for performance) plus a small capitation element. In 1989, the Scottish Home and Health Department stated; 'it is desirable that wherever possible the general dental services should provide care for the whole family, ${ }^{10}$ This was reiterated by the Scottish Office Department of Health which stated; 'the independent general dental practitioner (GDP) should remain the preferred provider of routine primary 
oral health care.'11 This 1997 guidance also stated that; "health boards have responsibility for monitoring availability of GDS.... if.... existing GDS provision is insufficient to meet the demand the health board may ask the Secretary of State, under Section 33 of the NHS (Scotland) Act 1978, to approve the appointment of a salaried dentist. "11

A recent strategic review of the dental workforce in Scotland reiterated the recommendation that the SGDS 'complement the current GDS. ${ }^{12}$

NHS primary care workforce data shows that since the 1997 guidance was issued, the number of salaried GDPs in Scotland has increased substantially. In March 1998, SGDS was $2.2 \%(41 / 1,913)$ of the workforce and GDS 97.8\% (1,872/1,913). By March 2010, the SGDS had risen to around $13 \%(376 / 2,884)$ and the GDS was $87 \%$ $(2,508 / 2,884)$. These data are reported to be complicated by double counting, partyear numbers and other inaccuracies; but nevertheless suggest a six-fold increase in the numbers of NHS salaried general dental services in Scotland over this 12-year period, while the total number of dentists increased by $50.5 \% .^{13}$

A previous report showed that between 1997 and 2003 the average SGDS dentist provided approximately $40 \%$ of the NHS treatments the average GDS dentist provided (note that private dental care was not included). ${ }^{14}$ This percentage has since been used as a correction factor in a Scottish Government report on access to dental care. ${ }^{15}$ However, the experience from pay-for-performance services in general medical practice suggests that a large proportion of this difference may simply be differential recording and reporting as a result of the financial incentives offered. ${ }^{16}$ Such incentives may influence GDS dentists but perhaps not SGDS dentists as they are not paid under a 'fee per item' system.

Similarly a review of primary care salaried dental service in Scotland from a reported SWOT analysis states that the SGDS is less cost effective than NHS (GDS) practice. ${ }^{17}$ It has also been stated that "policies to provide additional salaried dentists...would result in more dentists being needed overall...due to their lower activity rates. ${ }^{14}$ However, despite this finding, if a health board can show that "existing GDS provision is insufficient to meet the

Table 1 Dental registrations in Scotland by registration type and SIMD quintile for adults and children (data from ISD and GROS)

Children $(<18$ years $) \quad$ Adults $(>18$ years $)$

Population denominator

\begin{tabular}{|c|c|c|c|}
\hline \multirow{5}{*}{ 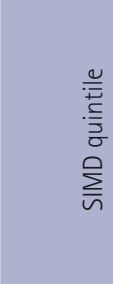 } & (deprived) 1 & 220,437 & 773,231 \\
\hline & 2 & 197,781 & 818,071 \\
\hline & 3 & 200,099 & 837,580 \\
\hline & 4 & 211,708 & 868,410 \\
\hline & (least-deprived) 5 & 212,122 & 854,561 \\
\hline \multicolumn{2}{|l|}{ Total } & $1,042,147$ & $4,151,853$ \\
\hline
\end{tabular}

GDS registrations

\begin{tabular}{|c|c|c|c|}
\hline \multirow{5}{*}{ 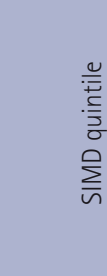 } & (deprived) 1 & 149,775 & 504,131 \\
\hline & 2 & 144,738 & 519,571 \\
\hline & 3 & 150,437 & 528,785 \\
\hline & 4 & 159,073 & 529,846 \\
\hline & (least-deprived) 5 & 165,249 & 535,654 \\
\hline \multicolumn{2}{|l|}{ Total } & 769,272 & $2,617,987$ \\
\hline \multicolumn{2}{|c|}{ RII $(95 \% \mathrm{CI})^{*}$} & 1.4 (0.4 to 2.5$)$ & $-0.1(-0.4$ to 0.1$)$ N.S. \\
\hline
\end{tabular}

\begin{tabular}{|c|c|c|c|}
\hline \multirow{5}{*}{ 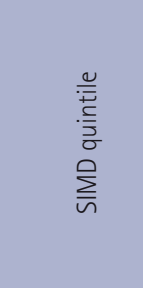 } & (deprived) 1 & 17,919 & 31,974 \\
\hline & 2 & 13,662 & 29,820 \\
\hline & 3 & 10,949 & 25,949 \\
\hline & 4 & 10,533 & 23,163 \\
\hline & (least-deprived) 5 & 9,137 & 21,298 \\
\hline \multicolumn{2}{|l|}{ Total } & 62,200 & 132,204 \\
\hline \multicolumn{2}{|l|}{ RII $(95 \% \mathrm{Cl})^{*}$} & $-7.8(-11$ to -4.8$)$ & $-1.6(-2.1$ to -1.1$)$ \\
\hline \multicolumn{4}{|c|}{ All dental registrations } \\
\hline \multirow{5}{*}{ 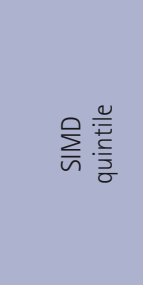 } & (deprived) 1 & 167,694 & 536,105 \\
\hline & 2 & 158,400 & 549,391 \\
\hline & 3 & 161,386 & 554,734 \\
\hline & 4 & 169,606 & 553,009 \\
\hline & (least-deprived) 5 & 174,386 & 556,952 \\
\hline \multicolumn{2}{|l|}{ Total } & 831,472 & $2,750,191$ \\
\hline \multicolumn{2}{|l|}{ RII $(95 \% \mathrm{Cl})^{*}$} & 0.8 (-0.7 to 1.5$)$ N.S. & $-0.2(-0.4$ to 0.0$)$ N.S \\
\hline
\end{tabular}

demand', then SGDS can be employed to provide dental care. ${ }^{11}$

This paper aims to use routine data to compare the socioeconomic distribution of patients registered with the salaried dental service with those registered with a nonsalaried service in an attempt to test the second part of Tudor Hart's thesis that the 'inverse care law operates more completely where medical care is most exposed to market forces, and less so where such exposure is reduced.'

\section{METHOD}

This is a retrospective cross-sectional secondary analysis of existing, anonymous data already in the public domain. Ethics approval was not required for the 


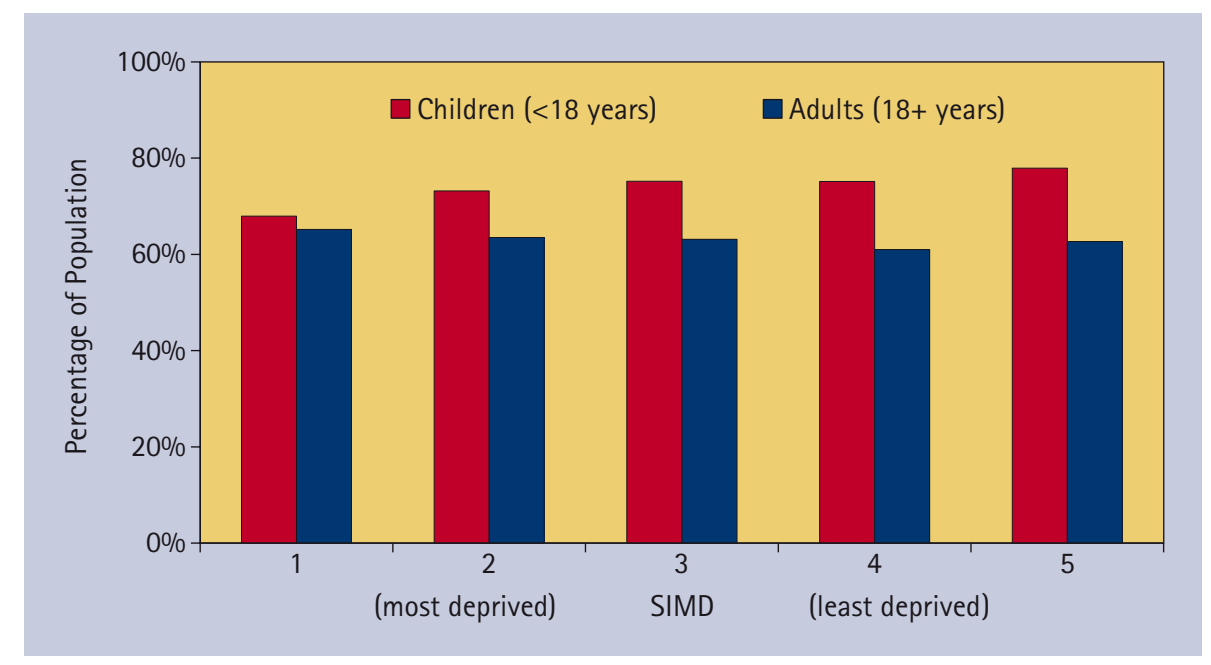

Fig. 1 NHS GDS registrations in Scotland by SIMD quintile of residence

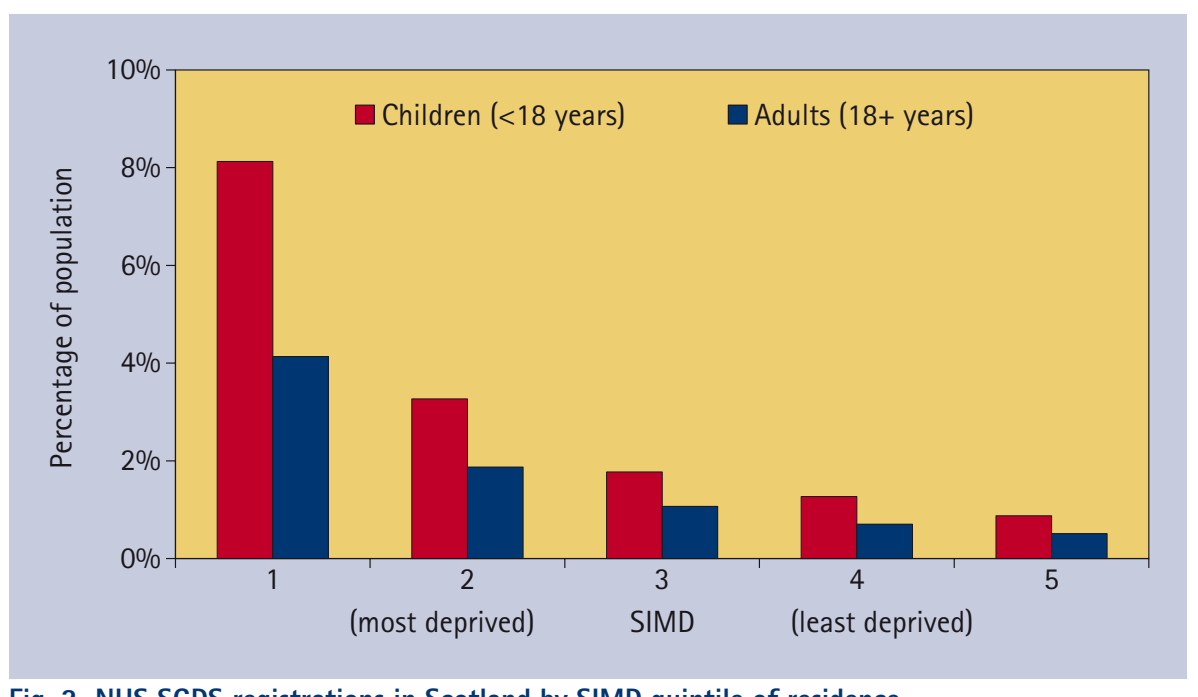

Fig. 2 NHS SGDS registrations in Scotland by SIMD quintile of residence

study. Routine administrative data were obtained from the Information Services Division (ISD) of NHS National Services Scotland. ${ }^{18}$ They included dental registrations for all ages and dichotomised into adults ( $>18$ years) and children $(<18$ years) by service of registration (non-salaried General Dental Services (GDS) or salaried General Dental Service (SGDS)) and Scottish Index of Multiple Deprivation (SIMD) quintile of area of residence. From these data, Spearman's rank correlation co-efficient and the relative index of inequality (RII) of dental registration were calculated. RIIs and the confidence intervals are very small numbers and are therefore given as multiples of $10^{-8}$.

The interpretation of RII is similar to the relative risk. For a linear distribution the regression based RII summarises the relative risk for the most advantaged group (SIMD quintile 5) compared to the least advantaged group (SIMD quintile 1). In this study a significant positive value indicates an inverse care law, whereas a negative value shows more deprived quintiles have higher registration rates. A statistically significant RII is indicated when the 95\% confidence interval does not include a value of zero.

\section{The Scottish Index of Multiple Deprivation (SIMD).}

The SIMD is assigned on the basis of the datazone (small area) of residence. Datazones are ranked by deprivation $(\mathrm{N}=6,505$; median of 769 people/datazone) and then stratified into quintiles. ${ }^{19}$ The SIMD classification has seven domains; income, employment, education, housing, health, crime and geographical access, which have been combined into an overall index to rank relative multiple deprivation.

\section{RESULTS}

The total number of NHS dental registrations in Scotland on 30 September 2010 was 3,581,663 (69\% of the total estimated Scottish population). Of those registered, the GDS had 3,387,259 (95\%) of the population and the SGDS had 194,404 (5\%) (see Table 1).

The RII which was statistically significant were NHS registrations for children in the GDS; NHS GDS registration is lower in children in deprived quintiles with a gradient $(\mathrm{RII}=1.4: 95 \%, \mathrm{CI} 0.4$ to 2.5, Spearman's $R=0.93, R^{2}=0.87$ ), see Figure 1.

In contrast, SGDS dental registration was much more common among those resident in deprived areas; being statistically significant for children (RII $=-7.8$ : 95\%, CI -11 to $\left.-4.8, \mathrm{R}=0.88, \mathrm{R}^{2}=0.77\right)$ and also for adults (RII $=-1.6: 95 \% \mathrm{CI},-2.1$ to -1.1 , $R=0.9, R^{2}=0.81$ ), see Figure 2 .

The combined impact of the two services in Scotland is that dental registrations show no overall social patterning.

\section{DISCUSSION}

Nine-five percent of the registered population in Scotland is with GDS. Five percent of the population is registered with the SGDS who made up 13\% of the total number of dentists. We were unable to identify any statistical test to apply to this data, but the finding broadly supports previous work that the average SGDS dentist provided approximately $40 \%$ of the NHS treatments the average GDS dentist provided. ${ }^{14}$

Dental registrations with SGDS dentists in Scotland are much more common among people living in the most deprived areas than in the least deprived areas, with a linear gradient across society. This is particularly the case for children. In contrast, dental registrations of adults with GDS dentists showed no consistent variation by deprivation.

In contrast to findings from previous reports on independent General Dental Services in England, ${ }^{2,7,8}$ those in Scotland did not display an inverse dental care law for adults although there was an inverse dental care law for children. However, when the registration number for both the GDS and the SGDS are combined, there was no evidence of an inverse dental care law.

The GDS is the preferred system of the Scottish Government for providing dental care to the population. ${ }^{10,11}$ The GDS was set up in 1948 and has been described as a 
system for paying dentists to provide treatment in the NHS, not as a public health measure..$^{20}$ The funding of the GDS is suggested as a contract between the public and the dentist. Therefore a public health measure aimed at reducing dental health inequalities is, according to the inverse dental care law, unlikely to be found in a market driven system such as the GDS.

The salaried and non-salaried dental services in Scotland clearly serve differing, but complementary population profiles. There may be several reasons for this. GDS practices are established as small businesses and the chosen location will depend on the business plan of the practice owner. Practices may not necessarily always be situated in areas of greatest need or provide the overall level of NHS provision to fully address local needs. In contrast, SGDS practice locations and sizes are planned to address unmet needs within the local area and should complement but not compete with GDS practices. The results of our study suggest that overall the two services are complementary in Scotland. Indeed, the architects of Dental Services in Scotland from the last decade should be recognised for trying to design an overall National dental service which goes some way to address the inverse dental care law.

Weaknesses in this study include no data on the provision of private dental care or mixed NHS/private care. Especially where there is a shortage of dentists to provide care, we speculate that it is likely a higher proportion of the population in the less deprived areas will seek private care as disposable income is likely to be greater, and there is no choice. Registration data has been criticised as it captures the demand rather than the need for dental care and is also produced as a by-product of the fee for item payment system. ${ }^{16}$

Levels of deprivation and dental disease are closely related. ${ }^{21-23}$ Dentists with a higher proportion of patients residing in the more deprived SIMD areas will therefore see individuals with, on average, more dental disease at presentation. Furthermore, the complexity (in terms of multiple morbidity and interacting social problems) is likely to be higher. This pattern is seen in general medical practice and it was expected to be similarly reflected in dentistry. ${ }^{24,25}$ Another factor not included in our analysis is the development of waiting lists for NHS dental treatment provided by the SGDS. Individuals from more deprived quintiles may be more likely to join an NHS waiting list and if lengthy, their treatment needs will have increased by the time care is provided.

The results from our study show that an inverse dental care law for NHS dental services does not operate in Scotland. The SGDS in Scotland is shown to have a higher proportion of registrations from those who live in more deprived areas. Continued planning and investment in both the GDS and in salaried services (SGDS), as a complementary service to the GDS, will ensure that current and future needs are met and consequently the inverse dental care law is tackled.

While great improvements have been made in the overall level of dental health in Scottish children, those in areas of higher deprivation continue to show higher dental disease levels, suggesting that dental health inequalities may not be improving. ${ }^{22,23}$ Our study suggests that where SGDS is provided for access to NHS dental care, they serve more deprived communities and will have continuing high demands placed upon it from patients with, on average, higher dental needs.

Further research is needed to clarify the reasons for the differences (that is, why people are registered with the SGDS, GDS and also private dentists). Possible reasons include migration, inability or unwillingness to pay privately, anxiety as they have lost touch with a former practice and SGDS being the only local option etc. Work on psychosocial impacts of these influences would also be useful to inform service design. We agree with Dyer et al. that the influence of private dental provision on the inverse dental care law should be considered in future studies. ${ }^{9}$ As private dental care is exposed to stronger market forces, we speculate that this will confirm an overall inverse dental care law for Scottish dentistry.
Competing interest: David Clouting is Clinical Director of Community Dental Services in NHS Borders. The authors report no other conflicts of interest.

1. Hart J T. The inverse care law. The Lancet 1971; 1: 405-412.

2. Jones $\mathrm{CM}$. Capitation registration and socia deprivation in England. An inverse 'dental' care law? Br Dent J 2001; 190: 203-206.

3. Cook P J, Walker R 0 . The geographical distribution of dental care in the United Kingdom.3. Regional synthesis and discussion Br Dent J 1967; 122: $551-558$

4. Scarrott D M. Changes in the regional distribution of general dental service manpower. Br Dent J 1978; 144: 359-363.

5. Buck D. Dental health, population size and the distribution of general dental practitioners in England. Community Dent Health 1999; 16: 149-153.

6. Carmichael C L. Inner city Britain: a challenge for the dental profession. Br Dent J 1985; 159: 24-27.

7. Landes D P, Adams G C, Whiston S. Distribution of NHS resources for children's dental care in general dental practice in England. Community Dent Health 2004; 21: 149-154.

8. Maunder P, Landes D P, Steen N. The equity of access to primary dental care for children in the North East of England. Community Dent Health 2006; 23: 116-119.

9. Dyer T A, Skinner J, Canning D, Green J R. A health equity methodology for auditing oral health and NHS General Dental Services in Sheffield, England. Community Dent Health 2010; 27: 68-73.

10. Scottish Home and Health Department. The National Health Service Community Dental Service. Publication no. DGM(89)15. Edinburgh: SHHD, 1989.

11. Scottish Office Health Department. Salaried and salaried plus bonus dentists providing GDS. Publication no. PCA(D) 10. Edinburgh: NHS SOHD, 1997.

12. NHS Education for Scotland. An analysis of the dental workforce in Scotland. A strategic review. Edinburgh: NES, 2010.

13. Information Services Devision ScotlandISD Website. http://www.isdscotlandarchive.scot.nhs.uk/isd/5345. html

14. NHS Education for Scotland. Workforce planning for dentistry in Scotland. Edinburgh: NES, 2004.

15. Newton T, Williams A, Bower E. Access to dental health services in Scotland. Edinburgh: Scottish Parliament Health Committee, 2nd report, 2005.

16. Gosden T, Forland F, Kristiansen I et al. Capitation, salary, fee-for service and mixed systems of payment: effects on the behaviour of primary care physicians. Cochrane Database Systc Rev2000; CD002215.

17. Taylor M. Review of primary care salaried dental services in Scotland. Edinburgh: Scottish Government, 2006.

18. ISD Website. http://www.isdscotland.org/HealthTopics/Dental-Care/Publications/data-tables. asp?id=705\#705

19. Scottish Index of Multiple Deprivation. 2009 general report. Edinburgh: Scottish Government National Statistics, 2009.

20. Kravitz T. GDSC Chairman looks forward, BDA News 1999; $12: 3$

21. Office for National Statistics. Adult dental health survey - oral health in the United Kingdom. Essex: HMSO, 1998.

22. Macpherson L M D, Conway D I, Goold S et al. National dental inspection programme of Scotland - report of the 2010 survey of $P 1$ children. Scottish Dental Epidemiology Coordinating Committee, 2010.

23. Marmot M, Bell R. Social determinants and dental health. Adv Dent Res 2011; 23: 201-206.

24. Watt $\mathrm{G}$. Patient encounters in very deprived areas. Br J Gen Pract 2011; 61: 146.

25. Watt G, Deep End Steering Group. GPs at the Deep End. Br J Gen Pract 2011; 61: 66-67. 